\title{
DESVIOS ROTACIONAIS NA FRATURA SUPRACONDILIANA DO ÚMERO EM CRIANÇAS: NOVO MÉTODO DE AVALIAÇÃO
}

\author{
ROTATIONAL DEVIATIONS IN SUPRACONDYLAR HUMERUS FRACTURES \\ IN CHILDREN: A NEW METHOD OF EVALUATION
}

Carlos Alberto Almeida de Assunção Filho', Rafael Parizzi Veloso', Miguel Akkari², Claudio Santilli ${ }^{3}$,

Carlos Alberto Almeida de Assunção ${ }^{4}$

\section{RESUMO}

Objetivo: Descrever um novo método para identificar e quantificar os desvios rotacionais da fratura supracondiliana do úmero. Métodos: O estudo foi realizado usando modelos sintéticos de úmero, serrando-o perpendicular ao seu longo eixo, na região da fossa olecraniana, fixando-o com diferentes desvios rotacionais, e então uma radiografia das peças foi obtida. Foi realizada uma análise descritiva da sensibilidade e especificidade do método de visualização do "esporão metafisário" com o teste não paramétrico de Kruskal-Wallis. A análise de regressão linear foi utilizada para identificar uma relação entre tamanho do esporão e o grau de desvio rotacional da fratura. Resultados: A sensibilidade e a especificidade do método foram de $100 \%$ a partir de 15 graus de desvio rotacional e houve uma relação estatisticamente significante entre o tamanho do esporão e o grau do desvio rotacional. Conclusão: O método demonstrou ser de fácil aplicação, confiável e reprodutível neste trabalho.

Descritores - Fraturas do Úmero; Cotovelo; Criança

\section{ABSTRACT}

Objective: To describe a new method to identify and quantify the rotational displacement on a supracondylar fracture of humerus. Methods: The study was conducted using synthetic models of the humerus, sawing it perpendicular to its long axis, in the olecranon fossa level, fixing with different rotational deviations, and then a radiograph of the construct was obtained. We performed a descriptive analysis of the sensitivity and specificity of the method of viewing the "metaphyseal spurs" with the nonparametric Kruskal- Wallis test. The linear regression analysis was used to identify a relationship between size of the spur and the degree of rotation deviation of the fracture. Results: The sensitivity and specificity was $100 \%$ from the 15 degrees of rotational deviation and there was a statistically relationship between the size of the spur and the degree of rotational deviation. Conclusion: The method proved to be easy to use, reliable and reproducible in this work.

Keywords - Humeral Fractures; Elbow; Child

\section{INTRODUÇÃO}

As fraturas do cotovelo correspondem cinco a $10 \%$ de todas as fraturas nas crianças ${ }^{(1)}$. Dentre estas, as fraturas supracondilianas do úmero são responsáveis por até 50 a $70 \%$ dos $\operatorname{casos}^{(2-4)}$, acometendo principalmente crianças na faixa etária entre cinco e sete anos de ida$\mathrm{de}^{(5)}$. As lesões ocorrem predominantemente à esquerda, ou do lado não dominante ${ }^{(5-7)}$.
O mecanismo de trauma mais comum é a queda com apoio do membro superior e o cotovelo em extensão completa, levando o olécrano a realizar um fulcro posterior na sua fossa, região de maior fragilidade, provocando uma fratura do tipo em extensão, isto é, com desvio posterior ${ }^{(8,9)}$. Este tipo de desvio representa cerca de $97 \%$ a $99 \%$ dos $\operatorname{casos}^{(5,8)}$.

Vários métodos de tratamento têm sido descritos,

1 - Residente do Departamento de Ortopedia e Traumatologia da Santa Casa de São Paulo - São Paulo, SP, Brasil.

2 - Doutor; Mestre do Departamento de Ortopedia e Traumatologia da Santa Casa de São Paulo - São Paulo, SP, Brasil.

3 - Professor Doutor da Faculdade de Ciências Médicas da Santa Casa de São Paulo - São Paulo, SP, Brasil.

4 - Médico Assistente do Departamento de Ortopedia e Traumatologia do Hospital Espanhol - Salvador, BA, Brasil.

Trabalho realizado no Departamento de Ortopedia e Traumatologia da Santa Casa de São Paulo.

Correspondência: Rua Marquês de Itu, 643, ap. 63, Vila Buarque - 01223-001 - São Paulo, SP. E-mail: assuncao.filho@yahoo.com.br

Trabalho recebido para publicação: 24/08/2011, aceito para publicação: 31/08/2011.

Os autores declaram inexistência de conflito de interesses na realização deste trabalho / The authors declare that there was no conflict of interest in conducting this work 
incluindo manipulação incruenta e imobilização em flexão $^{(10)}$, tração transesquelética ${ }^{(11)}$, redução fechada e fixação percutânea com fios de Kirschner ${ }^{(12)}$ e redução aberta e fixação interna ${ }^{(13)}$.

A complicação mais comum a longo prazo é o cúbito varo. Essa deformidade decorre principalmente de uma má redução inicial ou uma perda da redução no decorrer do tratamento ${ }^{(14-21)}$.

Habitualmente, durante a avaliação dos desvios da fratura, no momento da redução e durante o seguimento ambulatorial, são realizados exames radiográficos em duas posições (frente e perfil). Com estas incidências radiográficas pode-se identificar e quantificar desvios no plano frontal e sagital, por parâmetros já bem estabelecidos. Contudo, desvios rotacionais são mais difíceis de serem avaliados. Atribuem-se às más reduções rotacionais a ocorrência do cúbito varo, influenciando a perda da redução, devido à falta de apoio das colunas medial e lateral ${ }^{(9)}$.

O objetivo deste trabalho é descrever um novo método para identificar e quantificar os desvios rotacionais da fratura supracondiliana do úmero, que seja de fácil aplicabilidade e confiável com o uso de radiografias simples do cotovelo.

\section{MATERIAIS E MÉTODOS}

O estudo foi realizado usando modelos sintéticos de úmero, nos quais foi mimetizada uma fratura supra- condilar serrando-o perpendicular ao seu longo eixo, a $2 \mathrm{~cm}$ da superfície articular distal, na região da fossa olecraniana (Figura 1A e B).

Os fragmentos foram fixados com o auxílio de um fio de Kirschner de $1,5 \mathrm{~mm}$, com diferentes desvios rotacionais. Realizamos a rotação externa do fragmento distal em torno do eixo central do úmero, partindo da redução anatômica e evoluindo com variações a cada cinco graus, medida com auxílio de um goniômetro. Repetimos o procedimento cinco vezes para cada grau de rotação. Radiografamos as peças nas incidências anteroposterior (AP) e perfil (Figura 1C) em cada grau de rotação.

A conformidade anatômica regional do osso e a diferença da largura dos fragmentos proximal e distal, no nível do corte, produzida pelo desvio rotacional, resulta em uma imagem visualizada nas radiografias em perfil que denominamos de "esporão metafisário". Desta maneira, a identificação deste sinal sugere a existência de um desvio rotacional.

Para avaliação deste método selecionamos três séries de radiografias na incidência em perfil. Cada série era composta por cinco radiografias sem desvio comparadas com cinco radiografias com cada um dos desvios rotacionais. Na primeira série as radiografias com desvio tinham cinco graus; na segunda, 10 graus; e, na terceira, 15 graus.

Os grupos de radiografias obtidas foram analisadas
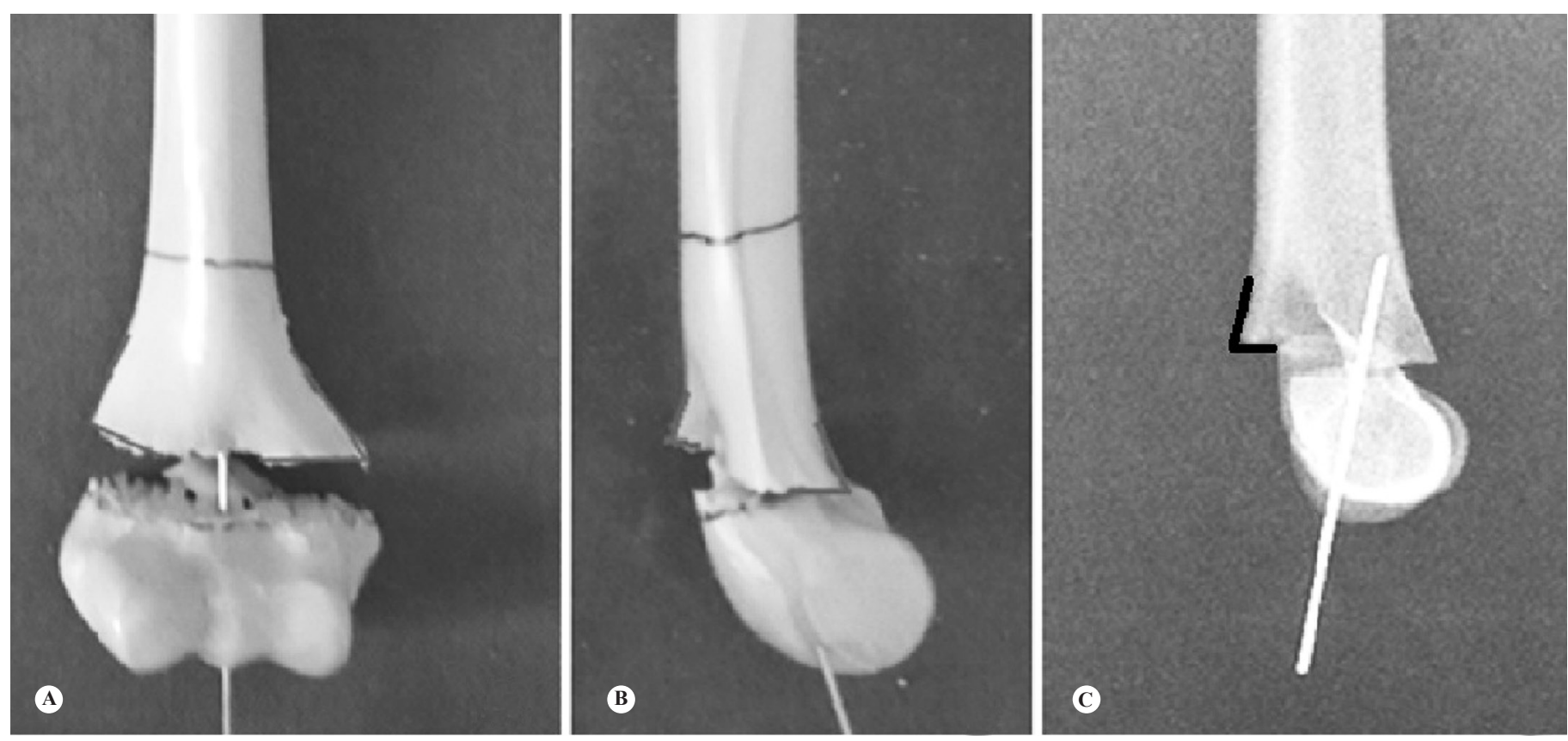

Figura 1 - Imagem e radiografia do "esporão metafisário" ( $A$ = visão de frente da fratura; $B$ = visão de perfil da fratura; e $C$ = radiografia de perfil evidenciando o desvio rotacional). 
por 55 médicos, sendo 23 ortopedistas de diversas especialidades, 13 residentes de ortopedia do terceiro ano, oito do segundo ano e 11 de primeiro ano, em uma reunião do Departamento de Ortopedia da Santa Casa de São Paulo. Fizemos uma breve explanação sobre a avaliação radiográfica de desvios rotacionais e o método proposto. As radiografias foram projetadas por cinco segundos cada. Após a exposição da radiografia, os observadores marcaram respostas positivas quando observassem o "esporão metafisário".

Mediante às respostas, foi realizada uma análise descritiva da sensibilidade e especificidade do método, para cada desvio rotacional. Para avaliar se houve diferença entre os grupos realizamos o teste não paramétrico de Kruskal-Wallis. Foi adotado um nível de significância de 5\%.

Objetivando encontrar uma relação entre o tamanho do "esporão" e o grau de desvio rotacional ao qual a "fratura" foi submetida, medimos as larguras dos fragmentos proximais e distais, imediatamente próxima à "fratura" foram medidas com auxílio da radiografia digital, pelo software Impax Results Viewer 1.0 da AGFA $^{\circledR}$ (Figura 2). Determinamos que o tamanho do "esporão" seria igual à diferença entre as larguras do fragmento proximal e do fragmento distal.

Para evitar vieses com a magnitude da radiografia, optamos por trabalhar com o percentual que o tamanho do "esporão" representava em relação à largura do fragmento distal, o qual deveria estar no seu perfil absoluto.

As medidas foram agrupadas em seis grupos, correspondentes ao desvio o qual a "fratura" foi submetida (zero, cinco, 10, 15, 20 e 25 graus). Realizamos uma avaliação estatística descritiva dos valores.

Utilizamos a análise de regressão linear para realizar uma relação entre a medida do "esporão metafisário" e o ângulo de desvio rotacional ao qual a "fratura" foi submetida.

\section{RESULTADOS}

As respostas mediante avaliação das séries radiográficas realizada pelos observadores nos permitiu calcular a sensibilidade e a especificidade do método para o reconhecimento de desvios rotacionais nos deferentes graus testados, como está disposto nas Tabelas 1, 2, 3 e 4 .

As análises estatísticas das medidas do percentual do esporão, agrupadas pelos graus de desvios os quais os fragmentos foram submetidos, estão representadas na Tabela 5.

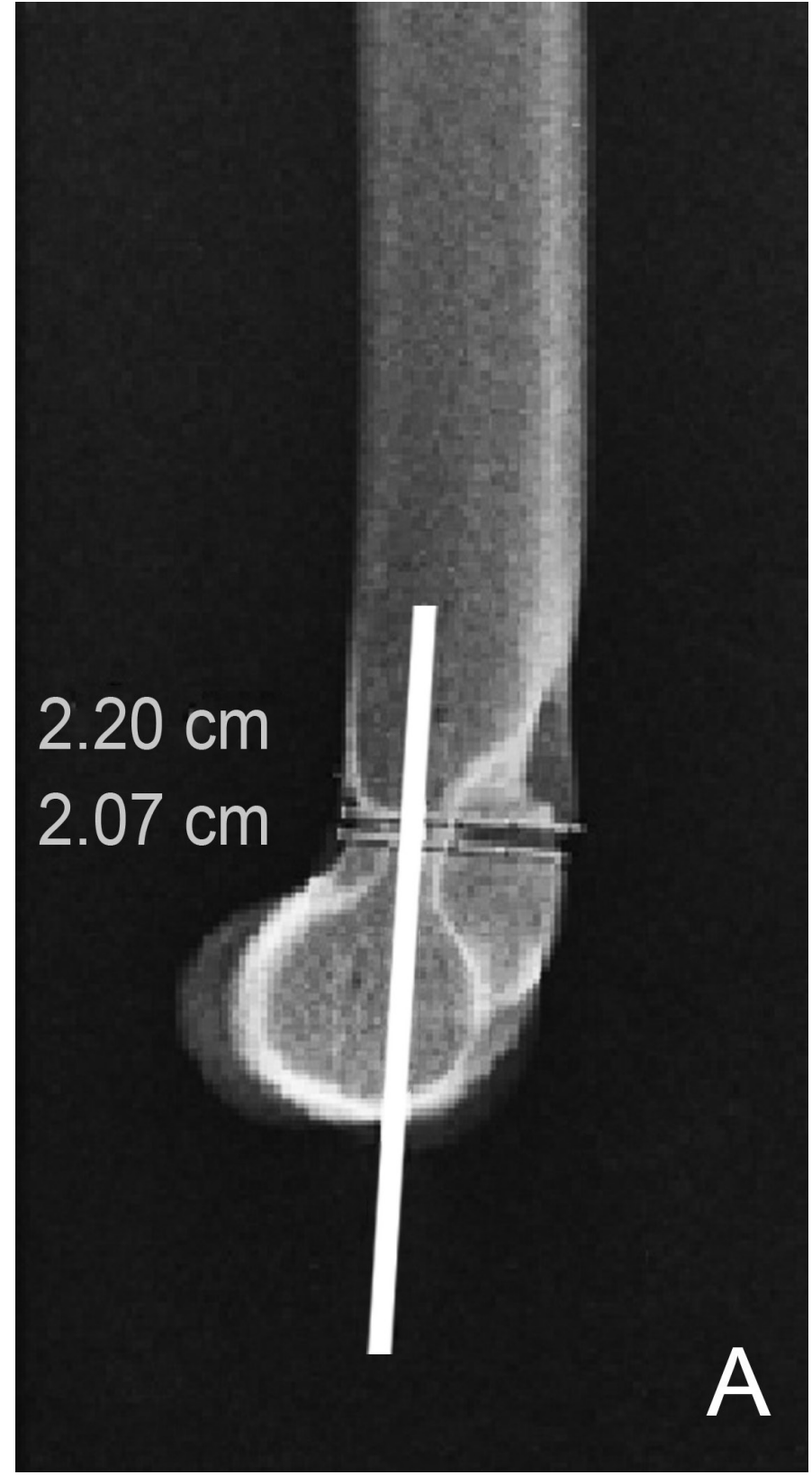

Figura 2 - Determinação das larguras dos fragmentos próximos à fratura pelo software Impax Results Viewer 1.0 AGFA (radiografia em perfil do modelo com cinco graus de desvio rotacional).

Tabela 1 - Sensibilidade do método do esporão metafisário nos desvios de cinco, 10 e 15 graus.

\begin{tabular}{|c|c|c|c|c|c|c|c|}
\hline \multicolumn{8}{|c|}{ Sensibilidade } \\
\hline Grau & $\mathbf{N}$ & Média & Mediana & $\begin{array}{l}\text { Desvio } \\
\text { padrão }\end{array}$ & Mínimo & Máximo & $p^{*}$ \\
\hline 5 & \multirow{3}{*}{55} & $80 \%$ & $80 \%$ & $19 \%$ & $40 \%$ & $100 \%$ & \multirow{3}{*}{$<0,001$} \\
\hline 10 & & $96 \%$ & $100 \%$ & $14 \%$ & $20 \%$ & $100 \%$ & \\
\hline 15 & & $100 \%$ & $100 \%$ & $0 \%$ & $100 \%$ & $100 \%$ & \\
\hline
\end{tabular}


Tabela 2 - Sensibilidade do método do esporão metafisário nos desvios de cinco, 10 e 15 graus para os diferentes grupos de observadores.

\begin{tabular}{|c|c|c|c|c|c|c|c|c|}
\hline $\begin{array}{c}\text { Sensibilidade } \\
\text { Grau }\end{array}$ & Grupo & $\mathbf{N}$ & Média & Mediana & Desvio padrão & Mínimo & Máximo & $\mathbf{p}^{*}$ \\
\hline \multirow{4}{*}{5} & Especialista & 23 & $76 \%$ & $80 \%$ & $22 \%$ & $40 \%$ & $100 \%$ & \multirow{4}{*}{0,48} \\
\hline & R3 & 13 & $83 \%$ & $80 \%$ & $18 \%$ & $60 \%$ & $100 \%$ & \\
\hline & $\mathrm{R} 2$ & 8 & $88 \%$ & $90 \%$ & $15 \%$ & $60 \%$ & $100 \%$ & \\
\hline & $\mathrm{R} 1$ & 11 & $78 \%$ & $80 \%$ & $17 \%$ & $60 \%$ & $100 \%$ & \\
\hline \multirow{4}{*}{10} & Especialista & 23 & $97 \%$ & $100 \%$ & $10 \%$ & $60 \%$ & $100 \%$ & \multirow{4}{*}{0,98} \\
\hline & R3 & 13 & $95 \%$ & $100 \%$ & $12 \%$ & $60 \%$ & $100 \%$ & \\
\hline & $\mathrm{R} 2$ & 8 & $98 \%$ & $100 \%$ & $7 \%$ & $80 \%$ & $100 \%$ & \\
\hline & $\mathrm{R} 1$ & 11 & $93 \%$ & $100 \%$ & $24 \%$ & $20 \%$ & $100 \%$ & \\
\hline \multirow{4}{*}{15} & Especialista & 23 & $100 \%$ & $100 \%$ & $0 \%$ & $100 \%$ & $100 \%$ & \multirow{4}{*}{1} \\
\hline & R3 & 13 & $100 \%$ & $100 \%$ & $0 \%$ & $100 \%$ & $100 \%$ & \\
\hline & $\mathrm{R} 2$ & 8 & $100 \%$ & $100 \%$ & $0 \%$ & $100 \%$ & $100 \%$ & \\
\hline & $\mathrm{R} 1$ & 11 & $100 \%$ & $100 \%$ & $0 \%$ & $100 \%$ & $100 \%$ & \\
\hline
\end{tabular}

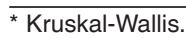

Tabela 3 - Especificidade do método do esporão metafisário nos desvios de cinco, 10 e 15 graus.

\begin{tabular}{|c|c|c|c|c|c|c|c|}
\hline \multicolumn{8}{|c|}{ Especificidade } \\
\hline Grau & $\mathbf{N}$ & Média & Mediana & Desvio padrão & Mínimo & Máximo & $\mathrm{p}^{*}$ \\
\hline 5 & \multirow{3}{*}{55} & $93 \%$ & $100 \%$ & $11 \%$ & $60 \%$ & $100 \%$ & \multirow{3}{*}{0,07} \\
\hline 10 & & $91 \%$ & $100 \%$ & $15 \%$ & $20 \%$ & $100 \%$ & \\
\hline 15 & & $88 \%$ & $80 \%$ & $14 \%$ & $40 \%$ & $100 \%$ & \\
\hline
\end{tabular}

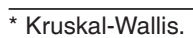

Tabela 4 - Especificidade do método do esporão metafisário nos desvios de cinco, 10 e 15 graus para os diferentes grupos de observadores.

\begin{tabular}{|c|c|c|c|c|c|c|c|c|}
\hline $\begin{array}{c}\text { Sensibilidade } \\
\text { Grau }\end{array}$ & Grupo & $\mathbf{N}$ & Média & Mediana & Desvio padrão & Mínimo & Máximo & $\mathbf{p}^{*}$ \\
\hline \multirow{4}{*}{5} & Especialista & 23 & $96 \%$ & $100 \%$ & $8 \%$ & $80 \%$ & $100 \%$ & \multirow{4}{*}{0,06} \\
\hline & R3 & 13 & $94 \%$ & $100 \%$ & $10 \%$ & $80 \%$ & $100 \%$ & \\
\hline & $\mathrm{R} 2$ & 8 & $95 \%$ & $100 \%$ & $14 \%$ & $60 \%$ & $100 \%$ & \\
\hline & $\mathrm{R} 1$ & 11 & $85 \%$ & $80 \%$ & $13 \%$ & $60 \%$ & $100 \%$ & \\
\hline \multirow{4}{*}{10} & Especialista & 23 & $94 \%$ & $100 \%$ & $9 \%$ & $80 \%$ & $100 \%$ & \multirow{4}{*}{0,5} \\
\hline & R3 & 13 & $91 \%$ & $100 \%$ & $13 \%$ & $60 \%$ & $100 \%$ & \\
\hline & $\mathrm{R} 2$ & 8 & $90 \%$ & $100 \%$ & $28 \%$ & $20 \%$ & $100 \%$ & \\
\hline & $\mathrm{R} 1$ & 11 & $87 \%$ & $100 \%$ & $16 \%$ & $60 \%$ & $100 \%$ & \\
\hline \multirow{4}{*}{15} & Especialista & 23 & $90 \%$ & $100 \%$ & $13 \%$ & $60 \%$ & $100 \%$ & \multirow{4}{*}{0,3} \\
\hline & R3 & 13 & $91 \%$ & $100 \%$ & $10 \%$ & $80 \%$ & $100 \%$ & \\
\hline & $\mathrm{R} 2$ & 8 & $78 \%$ & $80 \%$ & $20 \%$ & $40 \%$ & $100 \%$ & \\
\hline & $\mathrm{R} 1$ & 11 & $89 \%$ & $80 \%$ & $10 \%$ & $80 \%$ & $100 \%$ & \\
\hline
\end{tabular}

${ }^{*}$ Kruskal-Wallis.

Tabela 5 - Grau de desvio x esporão (\%) - análise descritiva.

\begin{tabular}{c|c|c|c|c|c}
\hline Grau & Media (\%) & Mediana (\%) & Desvio padrão & Mínimo & Máximo \\
\hline 0 & 0,82 & 0,44 & 0,99 & 3,00 & 3,20 \\
\hline 5 & 8,19 & 6,28 & 4,40 & 14,61 & 13,30 \\
\hline 10 & 18,06 & 16,28 & 4,68 & 25,81 & 36,19 \\
\hline 15 & 30,03 & 28,88 & 3,83 & 40,81 & 44,29 \\
\hline 20 & 42,62 & 43,33 & 1,53 & 47,64 & 53,78 \\
\hline
\end{tabular}


A análise de regressão linear que foi utilizada para identificar uma relação entre a percentagem do esporão e o grau de desvio rotacional da fratura determinou uma constante igual a 2,049, com $\mathrm{p}<0,001$.

A Figura 3 representa a dispersão dos valores da percentagem do "esporão" nos diferentes desvios rotacionais, assim como a reta regida pela fórmula determinada pela constante encontrada na análise de regressão linear.

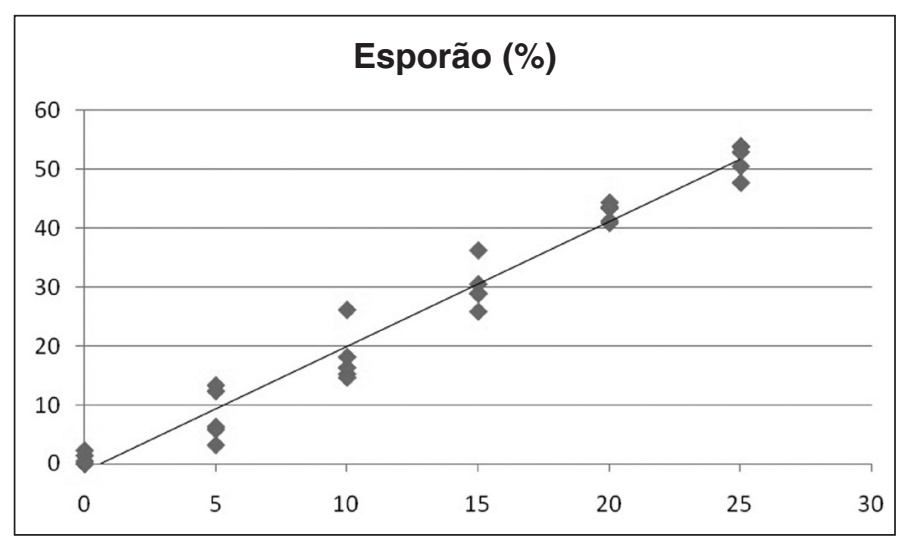

Figura 3 - Grau de desvio x esporão (\%) - gráfico de dispersão.

\section{DISCUSSÃO}

Nenhum método simples de avaliação dos desvios rotacionais da fratura supracondiliana do úmero foi popularizado $^{(22)}$. A diferença de largura dos fragmentos no nível da fratura é uma técnica bastante utilizada nas fraturas diafisárias, principalmente nas osteossínteses minimamente invasivas ${ }^{(23)}$.

Lonroth descreveu um método no qual várias radiografias são realizadas em intervalos de cinco graus de rotação da ampola em torno do local da fratura. As duas radiografias demonstrando maior dimensão nas linhas de fratura dos fragmentos proximal e distal são identificadas e a diferença entre os ângulos em que as radiografias foram tomadas é calculada e o desvio rotacional é estimado ${ }^{(24)}$. Gordon et al ${ }^{(25)}$ publicaram um método para estimar os desvios rotacionais que eles chamam de "percentagem de rotação". Usando uma visão lateral do cotovelo, o percentual de rotação lateral é calculado dividindo-se a dimensão da largura do fragmento proximal e do fragmento distal no local da fratura. O resultado é multiplicado por 100, produzindo a percentagem de rotação. Henderson et al demonstraram confiabilidade em um método matemático para aferir desvios rotacionais a partir das larguras dos fragmentos no local da fratura nas incidências anteroposterior (AP) e perfil e o cosseno do ângulo do desvio ${ }^{(22)}$.
Como exposto, os métodos já propostos na literatura não agrupam a praticidade, a confiabilidade e a possibilidade de realizá-los durante o procedimento cirúrgico.

Diante dos resultados de identificação de desvio rotacional, mediante a observação do esporão metafisário, podemos constatar que o método demonstrou ter boa sensibilidade, variando suas médias de 80 a 100\%. Houve uma melhora progressiva conforme o aumento do desvio rotacional, apresentando diferença estatisticamente significante $(\mathrm{p}<0,001)$, como mostra a Tabela 1. Fato este que pode ser explicado pelo aumento do tamanho do esporão nos desvios rotacionais maiores, tornando-se de mais fácil observação.

Observamos também que não houve diferença estatisticamente significante na sensibilidade do método entre os grupos de diferentes níveis de graduação, em todos os ângulos testados (Tabela 2), sugerindo que o método demonstrou ser de fácil compreensão e aplicabilidade, permitindo a todos, independente do grau de conhecimento e experiência, um bom desempenho.

Todos os observadores foram capazes de identificar o desvio rotacional, quando este era de 15 graus, através do método proposto. Desta maneira, acreditamos que trata-se de um método seguro, visto que a perda de apoio das colunas medial e lateral do terço distal do úmero e a perda da compensação do ombro se dá após os 30 graus de deformidade rotacional ${ }^{(22)}$.

A análise da especificidade do método demonstra bons resultados variando suas médias entre 78 e 96\%, o que sugere um baixo número de respostas falso-positivas. Houve uma tendência à queda da especificidade conforme o aumento da sensibilidade nos grupos de desvios rotacionais (Tabela 3); entretanto, não foi estatisticamente $(p=0,07)$. Também não tivemos diferença estatisticamente significante na análise da especificidade entre os grupos de diferentes níveis de orientação, o que demonstra novamente uma uniformidade das respostas entre os grupos.

A análise descritiva da percentagem do esporão nos permite concluir que houve uma variação pequena de seus valores dentro do mesmo grupo de desvio rotacional, com desvios padrão sempre abaixo da média, exceto para o grupo de zero grau que, por se tratar de valores muito baixos, resolvemos desconsiderar.

A observação do gráfico de dispersão da percentagem do esporão x grau de rotação demonstra uma progressão constante. Através da análise de regressão linear podemos afirmar que, para este estudo experimental, foi 
encontrada uma relação entre o desvio rotacional e a percentagem do "esporão metafisário". Sendo que esta regressão linear de maneira estatisticamente significante $(\mathrm{p}<0,001)$ determinou uma constante de relação entre as duas variáveis igual a 2,049. Desta maneira, esta relação pode ser regida pela seguinte fórmula: $Y=2,049$ * $\mathrm{X}$, onde $\mathrm{Y}$ representa a percentagem do tamanho do esporão em relação à largura do fragmento distal e X o valor em graus da deformidade rotacional.

Contudo, trata-se de um trabalho experimental no qual foi utilizado um osso sintético e a fratura foi reproduzida, o que pode diferir na avaliação de casos reais.

Durante a análise das radiografias na incidência em anteroposterior, constatamos que a diferença entre as larguras dos fragmentos era pequena, o que dificultou a avaliação. Desta maneira, optamos por trabalhar apenas com a incidência em perfil.

\section{CONCLUSÃO}

A observação do "esporão metafisário", representando diferença das larguras dos fragmentos e consequentemente desvio rotacional, demonstrou ser um método de fácil aplicação, confiável e reprodutível neste trabalho. Houve uma relação linear entre o tamanho do "esporão" e o grau de desvio rotacional, permitindo identificar o grau de desvio rotacional a partir das larguras dos fragmentos.

\section{REFERÊNCIAS}

1. Herring JA. Fractures about the elbow. In: Herring JA editor. Tachdjian's pediatric orthopaedics. 3rd ed. Philadelphia: W.B. Saunders Company; 2002. p. 2139-217.

2. Otsuka NY, Kasser JR. Supracondylar Fractures of the Humerus in Children. J Am Acad Orthop Surg. 1997;5(1):19-26.

3. Diméglio A. Growth in pediatric orthopaedics. In: Morrissy RT, Weinstein SL, editors. Lovell and Winter's pediatric orthopaedics. 6th ed. Philadelphia: Lippincott Williams and Wilkins; 2005. p 35-65.

4. Cheng JC, Ng BK, Ying SY, Lam PK. A 10-year study of the changes in the pattern and treatment of 6,493 fractures. J Pediatr Orthop. 1999;19(3):344-50.

5. Cheng JC, Lam TP, Maffulli N. Epidemiological features of supracondylar fractures of the humerus in Chinese children. J Pediatr Orthop B. 2001;10(1):63-7.

6. Farnsworth CL, Silva PD, Mubarak SJ. Etiology of supracondylar humerus fractures. J Pediatr Orthop. $1998 ; 18(1): 38-42$.

7. Topping RE, Blanco JS, Davis TJ. Clinical evaluation of crossed-pin versus lateral-pin fixation in displaced supracondylar humerus fractures. J Pediatr Orthop. 1995;15(4):435-9.

8. Mahan ST, May CD, Kocher MS. Operative management of displaced flexion supracondylar humerus fractures in children. J Pediatr Orthop. 2007;27(5):551-6.

9. Omid R, Choi PD, Skaggs DL. Supracondylar humeral fractures in children. J Bone Joint Surg Am. 2008;90(5):1121-32.

10. Siris IE. Supracondylar fracture of the humerus. An analysis of 330 cases. Surg Gynecol Obstet. 1939;68:201-22.

11. Graham HA. Supracondylar fractures of the elbow in children. Part II. Clin Orthop Relat Res. 1967;(54):93-102.

12. Kasser JR, Beaty JH. Supracondylar fractures of the distal humerus. In: Kasser JR, Beaty JH, editors. Rockwood and Willkins' fractures in children. 5th ed. Philadelphia: Lippincott Williams \& Willkins; 2001. p. 577-620.

13. Fowles JV, Kassab MT. Displaced supracondylar fractures of the elbow in children. A report on the fixation of extension and flexion fractures by two lateral percutaneous pins. J Bone Joint Surg Br. 1974;56(3):490-500.
14. Amspacher JC, Messenbaugh JF Jr. Supracondylar osteotomy of the humerus for correction of rotational and angular deformities of the elbow. South Med J. 1964;57:846-50.

15. Badhe NP, Howard PW. Olecranon screw traction for displaced supracondylar fractures of the humerus in children. Injury. 1998;29(6):457-60.

16. Mitchell WJ, Adams JP. Supracondylar fractures of the humerus in children: a ten-year review. JAMA. 1961;175:573-77.

17. Nacht JL, Ecker ML, Chung SM, Lotke PA, Das M. Supracondylar fractures of the humerus in children treated by closed reduction and percutaneous pinning. Clin Orthop Relat Res. 1983;(177):203-9.

18. Smith L. Deformity following supracondylar fractures of the humerus. J Boné Joint Surg Am. 1960;42:235-52.

19. Vahvanen V, Aalto K. Supracondylar fracture of the humerus in children. A long-term follow-up study of 107 cases. Acta Orthop Scand. 1978;49(3):225-33.

20. Weiland AJ, Meyer S, Tolo VT, Berg HL, Mueller J. Surgical treatment of displaced supracondylar fractures of the humerus in children. Analysis of fifty-two cases followed for five to fifteen years. J Bone Joint Surg Am. 1978;60(5):657-61.

21. Wilkins KE. Supracondylar fractures: what's new? J Pediatr Orthop B. 1997;6(2):110-6.

22. Henderson ER, Egol KA, van Bosse HJ, Schweitzer ME, Pettrone SK, Feldman DS. Calculation of rotational deformity in pediatric supracondylar humerus fractures. Skeletal Radiol. 2007r;36(3):229-35.

23. Krettek C, Miclau T, Grün O, Schandelmaier P, Tscherne H. Intraoperative control of axes, rotation and length in femoral and tibial fractures. Technical note. Injury. 1998;29(Suppl 3):C29-39.

24. Lonroth $\mathrm{H}$. Measurement of rotational displacement in supracondylar fractures of the humerus. Acta radiol. 1962;57:65-70.

25. Gordon JE, Patton CM, Luhmann SJ, Bassett GS, Schoenecker PL. Fracture stability after pinning of displaced supracondylar distal humerus fractures in children. J Pediatr Orthop. 2001;21(3):313-8. 